\title{
Latent discrimination learning under a regular schedule of partial reinforcement
}

\author{
E. J. CAPALDI \\ Purdue University, Lafayette, Indiana 47907
}

\begin{abstract}
In this investigation, which employed rats in a runway, discriminative responding consisted of faster running on the reinforced than on the nonreinforced trials of either the $4 \mathrm{NR}$ or R4N schedule, both schedules containing fixed, repeated sequences of nonreinforced and reinforced trials. Under the $4 \mathrm{NR}$ schedule, four nonreinforced trials preceded a reinforced trial each day, and under the $\mathrm{R} 4 \mathrm{~N}$ schedule, a reinforced trial was followed by four nonreinforced trials each day. The major finding obtained was that under the $4 \mathrm{NR}$ schedule, discriminative responding was improved very substantially by a shift to extinction. Rats maintained on the $4 \mathrm{NR}$ schedule did not show improved discriminative responding, nor did discriminative responding improve in extinction following training under either the $\mathrm{R} 4 \mathrm{~N}$ schedule or a schedule of consistent reinforcement. Latent discrimination learning was defined as discriminative responding which fails to reflect adequately the amount of discrimination learning accomplished. The present findings demonstrate latent discrimination learning for regular schedules of partial reinforcement, something already demonstrated for brightness differential conditioning and possibly DRL schedules, as well.
\end{abstract}

According to Spence (1936), stimuli which are reinforced gain in excitatory capacity to elicit reactions, while stimuli which are nonreinforced gain in capacity to inhibit reactions. Moreover, these excitatory and inhibitory tendencies generalize to other similar stimuli, and the more similar the stimuli, the greater the generalization. These discrimination learning postulates are accepted here. The present investigation was concerned with whether discrimination training which produces discrimination learning (as revealed by a subsequent test) is necessarily accompanied by discriminative responding during the initial training. Discrimination training which is not accompanied by discriminative responding may be explained in one of two general, but not necessarily incompatible, ways. The common or conventional approach is to assume a lack either of discrimination learning or some element necessary to it, attention to relevant cues (Krechevsky, 1932), or whatever, depending upon one's theory of discrimination learning (see, e.g., Sutherland \& Mackintosh, 1971). The second possibility is that discrimination learning has occurred that fails to reflect itself in discriminative responding, and that discrimination learning is, in effect, latent. Evidence presented by Capaldi, Berg, and Morris (1975) indicates that the variables responsible for latent discrimination learning may be numerous, largely undiscovered, and of more than one type, such as associative as well as motivational. Indeed, in the Capaldi et al. (1975) investigation, it was clear that

This research was supported in part by National Institute of Child Health and Development Grant HD 04379 to E. J. Capaldi. motivational and associative variables worked in tandem to mask the behavioral manifestation of the discrimination learning which had occurred. Thus, latent discrimination learning is defined here to include all cases where discrimination learning has occurred but is not manifested in performance. Latent discrimination learning can be due to a variety of variables, only a few of which, perhaps, can be presently identified (see Capaldi et al., 1975). The following set of conditions, utilized here, were considered adequate to provide evidence for latent discrimination learning: There is either an initial appearance or an improvement in discriminative responding, following a shift from a discrimination training procedure to another training procedure, such as extinction, which does not involve discrimination training and which could not, in itself, either initiate or sustain discriminative responding. Under the circumstances described, obviously, initiation or improvement in discriminative responding following the shift must be attributed to discrimination learning completed but not manifested before the shift, i.e., to latent discrimination learning.

The conditions described above were realized in two previous investigations (Capaldi et al., 1975; Ison \& Adinolfi, 1968). In those investigations, which employed quite minimal acquisition training, discriminative responding was absent in a differential conditioning situation employing parallel runways in which $100 \%$ reinforcement was associated with one brightness alternative and $0 \%$ with the other. The absence of discriminative responding in those investigations in the acquisition phase is not compatible with the hypothesis that too little training had been 
employed to produce discrimination learning, because on a shift to extinction, or $0 \%$ reinforcement, in both brightness alternatives, discriminative responding appeared promptly. This latent discrimination learning was shown by Capaldi et al. (1975) to be due to the presence of factors in the acquisition phase which normally mask discriminative responding early in acquisition training. These factors, in some cases at least, could be reduced or eliminated by the shift to extinction and so allow manifestation of discriminative responding in the extinction phase. A possibly related phenomenon was reported by Reynolds (1964). In that investigation, which employed a DRL schedule, the temporal discrimination established in acquisition showed improvement in extinction, resulting in the emergence in extinction of a finer discrimination of interresponse-time duration.

Latent discrimination learning was examined by employing a form of discrimination learning commonly called pattern learning. Pattern learning has been described (see Capaldi, 1967) as discrimination learning based on cues provided by a regular reinforcement schedule. An example of pattern learning is the faster reinforced-trial than nonreinforcedtrial speed which may ultimately occur when reinforced and nonreinforced trials are alternated, as in a runway (e.g., Campbell, Crumbaugh, Rhodus, \& Knouse, 1971; Capaldi, 1967; Tyler, Wortz, \& Bitterman, 1953). Single-alternation patterning occurs, according to one view (Capaldi, 1966; Capaldi $\&$ Morris, 1974), because a characteristic cue is produced by reinforcement (SR), as is by nonreinforcement $\left(\mathrm{SN}^{\mathrm{N}}\right)$, and under a single-alternating schedule, $\mathrm{SR}$ always occurs on nonreinforced trials and so is the $\mathrm{S}-$ cue, while $\mathrm{S}^{\mathrm{N}}$ always occurs on reinforced trials and so is the $\mathrm{S}+$ cue. According to this sort of analysis, so-called pattern learning is better described as a form of differential conditioning regulated by internal cues, or more briefly, internally cued differential conditioning.

In an investigation by Wike and King (1973), pattern responding begun in acquisition maintained itself over a considerable number of extinction trials. In our laboratory, recently, employing what is called the 4NR schedule (a regular schedule similar in some respects to one of those employed by Wike \& King), pattern running was not only maintained in extinction, but showed marked improvement. Of equal interest to us was that the improved discriminative responding that emerged in extinction and could have taken several forms took, in fact, a highly specific form, the exact form predicted by the sequential hypothesis (see, e.g., Capaldi, 1966, 1967), which in the case of the 4NR schedule is progressively increasing speed over the five trials of each day. Such progressively increasing speed was expected because of stimulus generalization. Under the $4 \mathrm{NR}$ schedule, each of the five trials of the day was accompanied by a distinctive stimulus (see, e.g., Capaldi, 1966). A similarity relation exists under the 4NR schedule, such that the stimulus that occurred on Trial 5 was more similar to that occurring on Trial 4 than to that occurring on Trial 3, and so on. Under the 4NR schedule, it was only the stimulus that occurred on Trial 5 of the day that was reinforced, all other stimuli being nonreinforced. Thus, the excitatory capacity directly acquired by the Trial 5 stimulus generalized less to the Trial 1 stimulus than to the Trial 2 stimulus, and so on. Accordingly, the sequential hypothesis predicted not merely discriminative responding under the 4NR schedule, but that speed should increase progressively over the five trials of each day. Since, under the 4NR schedule, this pattern of behavior was largely absent in acquisition but emerged clearly in extinction, it was suspected that the improved discriminative responding observed in extinction might represent a case of latent discrimination learning in the regular schedule partialreinforcement situation. This hypothesis was tested in this investigation, employing relevant control groups along with the 4 NR schedule.

Demonstrating latent discrimination learning in the regular schedule situation should have a number of implications. It should, for example, lend increased support to the view (see, e.g., Capaldi, et al., 1975) that partial reinforcement and conventional forms of discrimination learning are closely related. It should also provide a plausible basis for reconciling certain seemingly discrepant findings, to be considered later, concerned with the effects of partial reinforcement at long intertrial intervals. Finally, it should raise a number of additional questions concerning various forms of latent discrimination learning for, as will become clear, the latent discrimination learning examined here in the regular schedule situation appears far less amenable to reduction by increased discrimination training than does that examined in the brightness differential conditioning situation by Capaldi et al. (1975) and by Ison and Adinolfi (1968).

\section{METHOD}

\section{Subjects}

Subjects were 40 male albino rats, about 100 days old at the start of the experiment, purchased from the Holtzman Co., Madison, Wisconsin.

\footnotetext{
Apparatus

The straight-alley runway, which was painted gray and was covered with $1.3-\mathrm{cm}$ hardware cloth, was $208.4 \mathrm{~cm}$ long and $10.2 \mathrm{~cm}$ wide and had $22.9-\mathrm{cm}$ high sides. The startbox, $20.3 \mathrm{~cm}$ long, was separated from the remainder of the alley by a guillotine door which was raised about $3 \mathrm{sec}$ after the rat was placed in the startbox. Raising the guillotine door started the first of three clocks $(.01 \mathrm{sec})$. Interrupting a photobeam beyond the guillotine door stopped Clock 1 (start time) and started Clock 2. Clock 2 was stopped (run time) and Clock 3 started by interrupt-
} 
ing a photobeam $132.1 \mathrm{~cm}$ beyond the first. Clock 3 was stopped (goal time) by interrupting a photobeam $39.4 \mathrm{~cm}$ beyond the second photobeam and $6.4 \mathrm{~cm}$ in front of a brass $5.1 \times 10.2 \times$ $3.8 \mathrm{~cm}$ food cup. The brass cup contained a metal inset, $3.8 \times 3.2$ $\times 1.9 \mathrm{~cm}$, which held the food pellets on reinforced trials. A guillotine brass door confined the rat to the goal area. A solenoidoperated aluminum lid covered the food cup on all reinforced trials, preventing access to the pellets. This lid was recessed in the goal cup in such a manner that the rat could not determine prior to breaking the last photobeam if it was in the open or the closed position.

When the rat entered the goalbox on nonreinforced trials, the lid of the food cup was withdrawn, exposing clearly the empty food cup. On reinforced trials, the lid covering the food cup was in the closed position and it opened $10 \mathrm{sec}$ after the rat broke the last photobeam. On nonreinforced trials, the rat was confined to the goal area for $10 \mathrm{sec}$, and on reinforced trials, the rat was removed from the goalbox immediately after having eaten the last of the $1545-\mathrm{mg}$ pellets provided.

\section{Procedure}

Preliminary training. Upon arrival at the laboratory, the subjects were housed in individual cages with food and water being freely available. Deprivation, which consisted of $12 \mathrm{~g}$ /day of Wayne Lab Blox, began on Day 1 of pretraining. Water was always freely available. The subjects were divided into four groups of 10 rats each and were handled briefly on an individual basis on all pretraining days. On Days 10-12, the subjects received 15 $45-\mathrm{mg}$ Noyes pellets in the home cage. The number of pellets eaten either in the home cage or in the apparatus was always subtracted from the 12-g daily ration.

Experimental training. As indicated, on all reinforced trials there was a delay of $10 \mathrm{sec}$. There were five trials on each of the 30 days of experimental training. Over the first 20 days, Group CR was reinforced on all trials, with Groups 4NR-E, $4 N R-A$, and $R 4 N$ being reinforced on only one trial each day, all remaining trials being nonreinforced. For Groups 4 NR-E and 4 NR-A, the reinforced trial was always the last trial of the day, and for Group R4N, it was always the first trial of the day. Over the next 10 days, experimental extinction occurred for Groups CR, 4NR-E and R4N, with Group 4NR-A continuing to receive the $4 N R$ schedule, as on the first 20 days.

The rats were run in squads of eight, two from each of the four groups. A given squad was run at the same time each day, but the running order of the subjects within a squad was randomized daily. All rats in a squad received Trial $\mathrm{N}$ before any rat received Trial $N+1$, producing an intertrial interval of about $10 \mathrm{~min}$ in later acquisition training. The rat was allowed $60 \mathrm{sec}$ to complete any runway section. If more than $60 \mathrm{sec}$ was required, the time in excess of $60 \mathrm{sec}$ was added to the time required to complete the next alley section. If a rat failed to enter the goalbox within $180 \mathrm{sec}$, it was placed directly into the goalbox. Such placements were only rarely required, and even then, only later in extinction for Groups $\mathrm{Cr}$ and $\mathrm{R} 4 \mathrm{~N}$.

\section{RESULTS}

Figure 1 shows the run section speeds (centimeters/ second) on each of the five trials of each acquisition day in 5-day blocks for each of the three reinforcement schedules employed; Groups 4NR-E and 4NR-A did not differ and so the data for these groups were combined in Figure 1. The run section data provided in Figure 1 are entirely representative of those obtained in the start and goal sections.

Discriminative responding under either the $4 \mathrm{NR}$ or

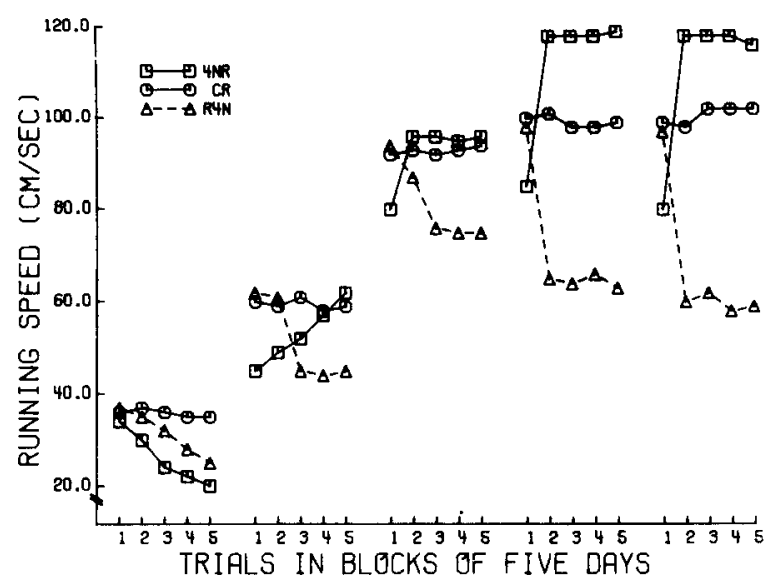

Figure 1. Run section speeds (centimeters/second) on each of the five trials of each acquisition day in 5-day blocks for each of the three reinforcement schedules employed.

R4N schedule occurs whenever running is slower on a nonreinforced trial than on the reinforced trial. Employing this criterion, it can be seen, In Figure 1, that by the last two blocks of trials discriminative responding was limited under the $4 \mathrm{NR}$ schedule and extensive under the R4N schedule. That is, while, by the end of training, running was slow on only one of the nonreinforced trials of the 4NR schedule, the first trial of the day, under the R4N schedule running on every nonreinforced trial was slower than on the reinforced trial. On the last two 5-day blocks of trials, differences among trials were significant under the $4 \mathrm{NR}$ schedule $[\mathrm{F}(4,36)=6.08, \mathrm{p}<.001]$ and under the $R 4 N$ schedule $[F(4,36)=6.27, p<.001]$. Subsequent Newman-Keuls tests indicated that under the 4NR schedule, Trial 1 speed differed from that on every other daily trial $(\mathrm{p}<.001)$, with no other difference being significant, and that under the R4N schedule, Trial 1 speed differed from that on every other trial $(p<.001)$, with no other difference being significant. On the last two blocks of trials, Groups 4NR-E and 4NR-A did not differ from each other on any of the daily trials (all $F s<1$ ).

As Figure 1 shows, beginning by about the third block of trials, faster running on the last four trials of the day occurred under the 4NR schedule than under the CR schedule, a difference which was significant on the last two blocks of trials $[F(1,28)$ $=8.75, \mathrm{p}<.01]$. On the second block of trials under the 4NR schedule, running was faster on the reinforced trial than on any nonreinforced trial. This difference was significant $[F(4,76)=3.89, p<.01]$, and Newman-Keuls tests indicated that, on the second block of trials under the 4NR schedule, running was faster on the reinforced trial than on any nonreinforced trial $(\mathrm{p}<.01)$ except the fourth of the day $(p>.05)$. Thus, under the 4NR schedule, the tendency toward discriminative responding was 
greater on the second block of trials than later in acquisition.

Other aspects of the early acquisition responding shown under the 4NR and R4N schedules are consistent with previous findings and require only brief comment. In prior investigations (Capaldi \& Hart, 1962; Capaldi, Turner, \& Wynn, 1962), it was reported that, in early acquisition under partial reinforcement, running speed declined progressively over the successive nonreinforced trials of the day. This tendency is clearly evident under both the 4NR and R4N schedules on the first blocks of trials. A second early acquisition trial tendency under partial reinforcement is for running to be faster following a reinforced than following a nonreinforced trial (see, e.g., Capaldi et al., 1975; Capaldi \& Hart, 1962; Tyler, et al, 1953). This tendency was quite evident under the R4N schedule. As can be seen in Figure 1, by as late as the third block of trials Group R4N ran more rapidly on Trial 2 of the day, a trial following reinforcement, than on any of the three subsequent trials of the days, all of which followed nonreinforcement.

Figure 2 shows, for Groups 4NR-E, CR, and R4N, which received extinction training, and for Group 4NR-A, which received continued acquisition training, running speed (centimeters/second) in the run section of the apparatus on each of the five trials in blocks of 2 days. As can be seen in Figure 2, Groups $C R$ and $R 4 N$ ran progressively more slowly and differed little in extinction, and with extinction training, the patterning manifested by Group R4N in acquisition decreased progressively and ultimately vanished.

In Group 4NR-A, the minimal sort of patterning begun earlier maintained itself without apparent improvement over additional acquisition trials. However, in Group 4NR-E, two trends are evident:

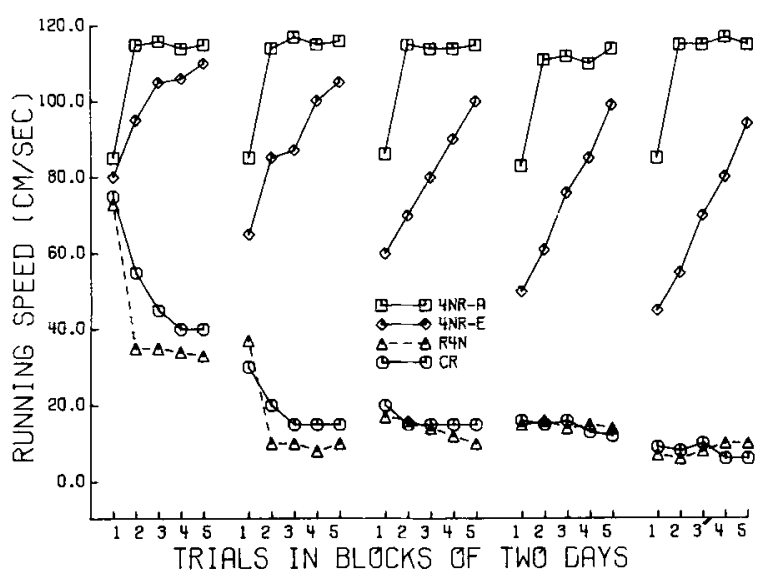

Figure 2. Run section speeds for Groups 4NR-E, CR, and R4N, which received extinction training, and for Group 4 NR-A, which received acquisition training on each of the five trials in blocks of 2 days.
Running speed declined over extinction days, and over extinction days pattern responding showed progressive improvement. Specifically, in Group 4NR-E there developed increasingly with extinction training the tendency for running speed to be slow on the first trial of the day and to increase progressively over the succeeding four daily trials. This tendency was even more marked than that shown on the second block of acquisition trials and, interestingly, while Group 4NR-E slowed down over extinction days, nevertheless, on the last block of extinction trials, Group 4NR-E ran about as rapidly on its formerly reinforced fifth trial of the day as Group CR had run on its reinforced trials in the later stages of acquisition (see Figure 1). Clearly, then, even by the end of extinction training, Group 4NR-E was running rapidly on its formerly reinforced acquisition trial. On the last block of extinction trials, the difference among trials was significant in Group 4NR-E [F $(4,36)$ $=9.22, \mathrm{p}<.001]$. Newman-Keuls tests indicated that Group 4NR-E ran faster on Trial 5, the reinforced trial in acquisition, than on Trial $4(p<.05)$ or on any other trial ( $\mathrm{ps}<.01$ at least).

\section{DISCUSSION}

In an investigation by Capaldi et al. (1975), which, as indicated, employed brightness differential conditioning, a group for which nonreinforced trials in $\mathrm{S}$ - were followed by reinforced trials in $\mathrm{S}+(\mathrm{N}-\mathrm{R}$ transitions) showed a lesser tendency toward discriminative responding than a group which did not receive $\mathrm{N}-\mathrm{R}$ transitions. The same, of course, was true here in the regular schedule situation, Group 4NR showing in the acquisition phase a smaller tendency toward discriminative responding than Group R4N. It might be noted, too, that increasing the magnitude of reinforcement increases discriminative responding in the differential conditioning brightness situation (e.g., Ludvigson \& Gay, 1966) and in the single alternation situation (Campbell et al., 1971; Capaldi, 1967).

Inconsistent with the hypothesis that improved discriminative responding by Group 4NR-E in extinction was due to extinction training itself, and independent of the type of acquisition training received, was the failure of either Group CR or Group R4N to show improved discriminative responding in extinction of the form shown by Group 4NR-E. Indeed, the failure of Group R4N to maintain its discriminative responding in extinction is inconsistent with still other alternatives to the latent discrimination view: that discriminative responding in extinction was due to having received in acquisition, either a low percentage of reinforcement, or any regular schedule of reinforcement, and so on. Moreover, the failure of additional training to improve discriminative re- 
sponding in Group 4NR-A suggests that extinction training reduced or eliminated the effectiveness of some factor or factors which produced latent discrimination learning under the 4NR schedule.

Explaining the present findings requires identifying the $\mathrm{S}+$ and $\mathrm{S}-$ cues regulating pattern responding. The stimuli assumed to be of major consequence are the distinctive cues associated with the first trial of the day or ST1 (Capaldi \& Morris, 1974), the internal cue produced by a reinforced trial, or $\mathrm{SR}$, and the internal cues produced by one, two, three, and four nonreinforced trials in a row, or $\mathrm{S}^{\mathrm{N}} 1, \mathrm{SN}^{\mathrm{N}}$, $\mathrm{S}^{\mathrm{N}} 3$, and $\mathrm{SN}^{\mathrm{N}}$, respectively (see, e.g., Capaldi 1966, 1967; Capaldi \& Morris, 1974). It is emphasized that ST1 is not on the same stimulus dimension as SR and the $S^{N}$ cues, and so is minimally similar to them, that $\mathrm{SR}^{\mathrm{R}}$ and the $\mathrm{SN}^{\mathrm{N}}$ cues are to some extent similar, and that the $\mathrm{S}^{\mathrm{N}}$ cues are highly similar. In the discrimination training phase, under the $\mathrm{R} 4 \mathrm{~N}$ schedule, running was slower on all nonreinforced trials than on the reinforced trials than on the reinforced trial, while under the $4 \mathrm{NR}$ schedule, only on one trial, the first of each day, was running slower on a nonreinforced than on the reinforced trial. In this sense, discriminative responding was better under the R4N schedule than under the 4NR schedule in the discrimination phase. Let us understand why this was so.

Under the R4N schedule, it was the cue associated with the first trial of the day, or ST1 that was reinforced, with the cues associated with each of the subsequent four trials of the day, or $\mathrm{SR}, \mathrm{SN} 1, \mathrm{SN}^{\mathrm{N}}$, and $\mathrm{SN}^{\mathrm{N}}$, respectively, being nonreinforced. The cue ST1 is only marginally similar to the cues SR, SNI, $\mathrm{SN}$, and $\mathrm{S} N$, and so these cues, which were always nonreinforced, received hardly any generalized associative strength from the reinforced $\mathrm{S}^{\mathrm{T} 1}$ cue. Because of this lack of stimulus generalization, it was expected that under the $\mathrm{R} 4 \mathrm{~N}$ schedule, discriminative responding would develop rapidly and that running would be slow and nondifferential on each of the daily four nonreinforced trials of the R4N schedule. This is exactly what occurred. Under the 4NR schedule, the cues occurring on the first four trials of the day, or $\mathrm{S}^{\mathrm{T} 1}, \mathrm{~S}^{\mathrm{N}} 1, \mathrm{~S}^{\mathrm{N}} 2$, and $\mathrm{S}^{\mathrm{N}} 3$, respectively, were nonreinforced, with $\mathrm{S}^{\mathrm{N}}$, the cue occurring on the last trial of the day, being reinforced. Thus, the $4 \mathrm{NR}$ schedule provides a relatively difficult discrimination problem, since three of the nonreinforced cues, $\mathrm{S}^{\mathrm{N}}$, $\mathrm{S}^{\mathrm{N} 2}$, and $\mathrm{S}^{\mathrm{N}}$, are highly similar to the reinforced $\mathrm{S}^{\mathrm{N} 4}$ cue, with only the $\mathrm{S}^{\mathrm{T} 1}$ cue, which was associated with slow responding, having little in common with the reinforced $\mathrm{SN}^{\mathrm{N}}$ cue. Associative strength directly acquired by $\mathrm{SN}^{\mathrm{N}}$ would, however, generalize in progressively decreasing fashion to the cues $\mathrm{SN}^{\mathrm{N}}, \mathrm{S}^{\mathrm{N}} 2$, $\mathrm{S}^{\mathrm{N} 1}$, and $\mathrm{S}^{\mathrm{T} 1}$, and so the present view explains the specific form of discriminative responding produced by the $4 \mathrm{NR}$ schedule early in acquisition and later in extinction, progressively increasing speed over the five trials of the day. The question for the present approach is, then, why did latent discrimination learning occur under the $4 \mathrm{NR}$ schedule?

It will be recalled that late in acquisition, except for Trial 1 of each day, faster running was associated with the 4NR schedule than with the CR schedule, rather direct evidence of an unusually strong tendency to respond under the 4NR schedule. Faster running under partial than under consistent reinforcement is called the partial reinforcement acquisition effect, or PRAE (see, e.g., Capaldi, 1974). According to Capaldi (1974), the basis of the PRAE is associative; in this specific instance, the PRAE occurrence because, under the 4NR schedule, the stimulus $\mathrm{S}^{\mathrm{N}} 4$ acquired a very strong capacity to elicit the instrumental reaction. Why this is so was explained by Capaldi (1974) and need not concern us here. The point we wish to emphasize here is that where, as in this investigation, an extraordinary amount of associative strength is acquired by $\mathrm{S}^{\mathrm{N}}$, then the generalized associative strength supplied by $\mathrm{S}^{\mathrm{N}} 4$ to $\mathrm{SN}^{\mathrm{N}}, \mathrm{S}^{\mathrm{N}} 2$, and $\mathrm{SN} 1$, but not, of course, to $\mathrm{ST}^{\mathrm{T}}$, might be great enough to evoke the reaction strongly, indeed, asymptotically. Where this is the case, differences in associative strength among the cues $\mathrm{SN}^{\mathrm{N}}, \mathrm{SN} 3, \mathrm{SN} 2$, and $\mathrm{SN} 1$, even if quite large, would nevertheless be irrelevant in terms of promoting discriminative responding, because, in effect, the upper limit of running speed or the ceiling would have been reached. But, according to this view, discriminative responding under the 4NR schedule would occur where associative strength to $\mathrm{S} N 4$ was relatively weak, such as early in acquisition (the growth of associative strength was not yet completed) or later in extinction (associative strength reduced by extinction training).

The demonstration of latent discrimination learning here provides a basis for reconciling certain discrepant partial reinforcement findings. Under a long intertrial interval such as $24 \mathrm{~h}$, single alternation (SA) training has often failed to produce SA patterning. The conventional interpretation of this failure essentially takes for granted that a failure of SA pattern responding means a failure of SA discrimination learning, something which, as the findings obtained here show, may not necessarily be the case. Specifically, it has been suggested that the cues SR and $\mathrm{S}^{\mathrm{N}}$ are fading traces which are available to serve as the $\mathrm{S}$ - and $\mathrm{S}+$ cue, respectively, at massed trials but not at spaced trials (see, e.g., Katz, Woods, \& Carrithers, 1966; Surridge \& Amsel, 1965). There are two difficulties with this view. First, SA patterning is sometimes obtained at a 24 -h intertrial interval (e.g., Capaldi \& Lynch, 1966; Jobe, Mellgren, Feinberg, Littlejohn, \& Rigby, 1977). Second, a variety of extinction data indicate that $S R$ and $S N$ are functional at a long as well as at a short inter- 
trial interval (e.g., Capaldi \& Capaldi, 1970; Capaldi \& Morris, 1976; Mellgren \& Seybert, 1973). A hypothesis which resolves these discrepant findings is that $S R$ and $S N$ are functional at all intertrial intervals, but at long intertrial intervals, latent discrimination learning is likely under an SA schedule; certainly factors must be optimal at a long intertrial interval to obtain SA patterning (see Jobe et al., 1977). Previous data aside, note that the assumption that $\mathrm{SN}^{\mathrm{N}}$ is a rapidly fading trace will not serve to explain the present patterning findings in extinction. Recall that, in extinction, Group 4NR-E showed progressively increasing speeds over the five trials of each day. A minimum intertrial interval of $10 \mathrm{~min}$ was employed here. Thus, in Group 4NR-E, at least 40 min elapsed between the termination of the first trial of each day and the initiation of the last trial of each day. Thus, in Group 4NR-E, information stored on the first trial of each day must have been available in some form as much as $40 \mathrm{~min}$ later, or else progressive improvement over trials would not have occurred.

The latent discrimination learning observed in the brightness differential conditioning situation (Capaldi et al., 1975; Ison \& Adinolfi, 1968) was associated with early acquisition trials and clearly was such that additional discrimination training would have produced improved discriminative responding. That is, discriminative responding that would have appeared in due course was facilitated by extinction training. This does not seem to have been the case here. That is, it appears that, in this investigation, extinction training was necessary for improved discriminative responding, and that, in effect, no additional amount of training under the 4NR schedule would have produced improved discriminative responding. Thus, there appear to be at least two forms of latent discrimination learning, a more of less temporary form and a more or less permanent form. The word permanent refers to the inability or relative inability of discrimination training (not extinction) to produce discriminative responding. In any case, the explanation provided here for permanent latent discrimination learning differs considerably from that provided for temporary latent discrimination learning (see Capaldi et al., 1975), a circumstance that reflects the present view that a wide variety of factors may be capable of producing latent discrimination learning.

\section{REFERENCES}

Campbell, P. E., Crumbaugh, C. M., Rhodus, D. M., \& KNouse, S. B. Magnitude of partial reward and amount of training in the rat: An hypothesis of sequential effects. Journal of Comparative and Physiological Psychology, 1971, 75, 120-128.

CAPALDI, E. J. Partial reinforcement: A hypothesis of sequential effects. Psychological Review, 1966, 73, 459-477.

Capaldi, E. J. A sequential hypothesis of instrumental learning. In K. W. Spence \& J. T. Spence (Eds.), The psychology of learning and motivation (Vol. 1). New York: Academic Press, 1967.

Capald, E. J. Partial reward either following or preceding consistent reward: A case of reinforcement level. Journal of Experimental Psychology, 1974, 102, 954-962.

Capaldi, E. J., Berg, R., \& Morris, M. D. Stimulus control of responding in the early trials of differential conditioning. Learning and Motivation, 1975, 6, 217-229.

Capaldi, E. J., \& Capaldi, E. D. Magnitude of partial reward, irregular reward schedules and a 24-hr ITI: A test of several hypotheses. Journal of Comparative and Physiological Psychology, 1970, 72, 203-209.

CAPALDI, E. J., \& HART, D. Influence of small number of partial reinforced trials on resistance to extinction. Journal of Experimental Psychology, 1962, 64, 166-171.

CAPAldi, E. J., \& LYNCH, D. Patterning at a 24-hour ITI: Resolution of a discrepancy more apparent than real. Psychonomic Science, 1966, 6, 229-230.

CAPAldi, E. J., \&-MorRis, M. D. Reward schedule effects in extinction: Intertrial interval, memory and memory retrieval. Learning and Motivation, 1974, 5, 473-483.

CAPAldi, E. J., \& Morris, M. D. A role of stimulus compounds in eliciting responses: Relatively spaced extinction following massed acquisition. Animal Learning \& Behavior, 1976, 4, 113-117.

Capaldi, E. J., Turner, L., \& Wynn, W. H. Decremental and facilitative effects in the straight-alley runway under partial reinforcement. Journal of Comparative and Physiological Psychology, 1962, 55, 545-549.

Ison, J. R., \& ADINolfi, A. A. Differential resistance to extinction determined by a small number of differential instrumental conditioning trials. Journal of Experimental Psychology, 1968, 77, 350-352.

Jobe, J. B., Mellgren, R. L., Feingerg, R. A., Littilejohn, R. L., \& RIGBY, R. L. Patterning, partial reinforcement and $\mathbf{N}$-length effects at spaced triais as a function of reinforcement of retrieval cues. Learning and Motivation, 1977, 8, 77-97.

Katz, S., Woods, G. T., \& Carrithers, J. H. Reinforcement aftereffects and intertrial interval. Journal of Experimental Psychology, 1966, 72, 624-626.

Krechevsky, I. Hypotheses in rats. Psychological Review, 1932, $39,516-532$.

Ludvigson, H. W., \& Gay, S. E. Differential reward conditioning: $\mathrm{S}-$ contrast as a function of the magnitude of S +. Psychonomic Science, 1966, 5, 289-290.

Mellgren, R. L., \& Seybert, J. A. Resistance to extinction at spaced trials using the within-subject procedure. Journal of Experimental Psychology, 1973, 100, 151-157.

REYNolds, G. S. Temporally spaced responding by pigeons: Development and effects of deprivation and extinction. Journal of the Experimental Analysis of Behavior, 1964, 1, 415-421.

SPENCE, $K$. W. The nature of discrimination learning in animals. Psychological Review, 1936, 43, 427-449.

Surridge, C. T., \& Amsel, A. Performance under a single alternation schedule of reinforcement at 24-hour intertrial interval. Psychonomic Science, 1965, 3, 131-132.

Sutherland, N. S., \& Mackintosh, N. J. Mechanisms of animal discrimination learning. New York: Academic Press, 1971.

Tyler, D. W., Wortz, E. C., \& Bitterman, M. E. The effect of random and alternating reinforcement on resistance to extinction in the rat. American Journal of Psychology, 1953, 66, 57-65.

WIKE, E. L., \& KING, D. D. Sequences of reward magnitude and runway performance. Animal Learning \& Behavior, 1973, 1, 175-178.

(Received for publication October 17, 1977; revision accepted August 22, 1978.) 